\title{
Comparison of Recombinant Human Bone Morphogenetic Protein-2 versus Iliac Crest Autograft in Anterior Cervical Discectomy and Fusion
}

\section{Jinhui Shi}

First Affiliated Hospital of Soochow University

Yonghui Huang

Affiliated Hospital of Jiangsu University

\section{Sheng Song}

Wuxi No.9 People's Hospital

\section{Feng Yuan}

The Affiliated Hospital of Xuzhou Medical University

\section{Gang Liu}

Jinling Hospital, Nanjing University

Jian Bian

Jiangsu Taizhou People's Hospital

\section{Meng Si}

Qilu Hospital of Shandong University

\section{Feng Zhou}

First Affiliated Hospital of Soochow University

\section{Xuesong Zhu}

First Affiliated Hospital of Soochow University

\section{Huilin Yang}

First Affiliated Hospital of Soochow University

Weimin Jiang ( $\nabla$ jwm_suzhou@126.com )

First Affiliated Hospital of Soochow University https://orcid.org/0000-0001-6206-7719

\section{Research article}

Keywords: anterior cervical discectomy and fusion, bone graft, dysphagia, fusion, rhBMP-2

Posted Date: May 29th, 2020

DOI: https://doi.org/10.21203/rs.3.rs-28301/v1 
License: (c) (i) This work is licensed under a Creative Commons Attribution 4.0 International License. Read Full License 


\section{Abstract}

\section{Backgroud}

The use of rhBMP-2 in anterior cervical discectomy and fusion (ACDF) is still "off-label" therapy because of higher incidence of potential complications such as adverse swelling, dysphagia, and airway compromise. The purpose of this study is to investigate whether the low dose rhBMP-2 $(0.5 \mathrm{mg} / \mathrm{level})$ decreases the incidence and severity of local complications in ACDF.

Methods

173 consecutively patients from June 2015 to March 2016 at seven participating centers in the China were enrolled. All the patients had primary one- or two-level ACDF with either rhBMP-2(BMP, $n=110)$ or iliac-crest bone autograft (IGB, $n=63$ ) were analyzed. Patients in both groups had compared preoperative pain and disability. The follow up timing was postoperative third days, postoperative-1, 3, 6 and 12 months.

Results

Patient demographics have no significantly difference between the BMP group and IGB group preoperation $(P>0.05)$. No significant difference in overall dysphagia incidence between the two groups $(p>$ 0.05). There was significant difference of PSTS at C3 level between two groups at all interval time $(P<0.01)$. There was significant difference of PSTS at $C 6$ level at postoperative third days $(P<0.01)$ and 3 months (0.010.05). No pseudarthrosis in both Groups. No significant difference in VAS scores, JOA and NDI .scores between two groups $(P>0.05)$.

Conclusion

The adverse events associated with low dose rhBMP-2 for one- and two-level ACDF are rare. We recommend placing the rhBMP-2 carrier in the center of cage, and bilateral coverage of the carrier with excised local osteophytes.

\section{Background}

Bone morphogenetic proteins (BMPs) are one of the most well-known growth factors which have the ability to induce the formation of bone and cartilage. In 1988, Wozney et al [1] cloned the human BMP gene sequence and expressed recombinant human bone morphogenetic protein-2 (rhBMP-2) by gene recombination technology. rhBMP-2 can induce: the differentiation of mesenchymal stem cells into chondrocytes and bone cells; participate in the process of bone and cartilage development and reconstruction; and accelerate the repair of bone defects and the occurrence of bone fusion[1, 2]. In 2002, the United States Food and Drug Administration (FDA) approved recombinant human bone morphogenetic protein for use in single-level anterior lumbar interbody fusion procedures. A large number of studies have shown that rhBMP-2 has a strong osteogenic ability and can increase spinal fusion 
rates $[3,4]$. Therefore, it is widely used "off-label" to eliminate the traditional need for harvesting of the iliac crest autograft. Unfortunately, clinical studies have found that the application of rhBMP-2 to anterior cervical discectomy and fusion (ACDF) can cause severe prevertebral soft tissue swelling, dysphagia, and even airway obstruction[5-7]. In 2008, the FDA issued a warning that the use of rhBMP in anterior cervical surgery may lead to life-threatening complications. However, the immediate and longterm morbidity associated with iliac crest harvest as autogenous bone grafts in ACDF is well-recognized. Using allograft bone has shown greater nonunion rates, delayed unions, and higher rates of graft subsidence[8-10]. At this time, the benefits and risks of using rhBMP-2 in ACDF is still controversial. The aim of this study was to compare the efficacy and complications between rhBMP-2 and iliac crest bone graft as well as to investigate whether the low dose rhBMP-2 $(0.5 \mathrm{mg} / \mathrm{level})$ decreases the incidence and severity of local complications when used in one- or two-level ACDF.

\section{Methods}

\subsection{Study Design}

The study was designed as a multicenter, prospective and controlled study, to optimize the carrier, dosage and implantation method of rhBMP-2 for anterior cervical fusion. The protocol was approved by the committee on the ethics of our University. All patients fully understand the benefits and risks of clinical trials and sign informed consent documents. This study included 173 patients who underwent one- or two-level ACDF procedures from June 2015 to March 2016 at seven participating centers in China. The inclusion criteria were adult patients (18-75 years old) whose primary diagnosis was as follows: cervical spondylotic myelopathy (CSM), cervical spondylosis radiculopathy (CSR), central cord syndrome (CCS). The exclusion criteria were: infection, tumor, allergy, heart failure, hepatic or renal dysfunction, pregnancy and lactation women. Figure 1 gives flowchart of patient selections. Hospital records were reviewed, demographic data on the patients were recorded, including age, sex, smoking status, primary diagnosis, fusion level, operation time and segment, duration of symptoms, estimated blood loss, length of stay, and complications.

Each center randomly assigned patients to the rhBMP-2 graft group (BMP) or iliac crest bone graft group (IBG) according to their own share. Operations were performed by a single spine surgeon who was experienced in performing ACDF procedures in each participating spine centers. The implantation used for ACDF included two types: PEEK cage plus anterior cervical plate; or a new stand-alone, zero profile anchored spacers PEEK cage (ROI-C, LDR, Troyes, France). In the BMP group, 0.5mg rhBMP-2 (Huadong Medicine Co. Ltd, Hangzhou China) was administered per level in the center of a PEEK cage in ACDF procedure, and peripheral of the carrier were packed with bone shavings and any resected anterior osteophytes obtained from decompression. The carrier material of rhBMP-2 was medicinal gelatin, injection grade lecithin and hydroxyapatite. (Figure 2) In IBG group, the PEEK cage was filled with autograft cancellous bone. All patient in both groups were also administered intravenous $1.0 \mathrm{~g}$ of Methylprednisolone injection intraoperatively and given another $80 \mathrm{mg}$ dose as well as $20 \%$ mannitol for 72 hours postoperatively. Then, patients were discharged home. 
The clinical outcome and complications of the BMP and IBG groups were compared at the time of followups. The postoperative follow-ups were: the third day postoperative, 1-month, 3-month, 6-month and 12month postoperative follow-ups. The neck disability index (NDI) scores questionnaire and Japanese Orthopedic Association (JOA囚scores questionnaire were used to evaluate the clinical outcomes for both groups. The incidence of postoperative dysphagia was recorded using a modified version of the Dysphagia Scoring System[11-14].

\subsection{Radiological Analysis}

Standing lateral radiography was used to measure prevertebral soft tissue swelling (PSTS). This lateral radiography obtained the anterior and posterior diameters of the anterior soft tissue for $\mathrm{C} 3$ (for the pharyngeal airway) and C6 (for the laryngeal airway). Picture Archiving and Communication System (PACS, Siemens Magic Software, Germany) was used to measure the distance from the anterior cortex in the mid-section of each vertebral body horizontally to the spot where the shadow of the airway can be seen [15]. (Figure 3)

Fusion results were evaluated using flexion-extension radiographs obtained at 3, 6 and 12 months after surgery. Axial CT scans of the cervical spine with sagittal and coronal CT reconstructions were obtained at 3 months to confirm fusion. In cases where fusion was uncertain at 3 months, another CT scan was obtained at 6 months or at 12 months if fusion was still not solid. Solid intervertebral fusion was considered on the basis of the following criteria[16-18]: (1) a continuous bone bridge is seen between the intervertebral bodies in X-ray; (2) no movement of the fusion segment on the flexion-extension lateral Xray. (3) 3D-reconstruction CT scans showed the presence of bridging bone and/or the lack of radiolucency at the graft-vertebral junction in both the coronal and sagittal planes.

\subsection{Statistical Analysis}

Statistical analysis was performed using SPSS (version 22.0, Chicago, IL, USA). Continuous variables were expressed as mean \pm standard deviation. Comparisons were made between the two groups using a two-sample $t$ test for continuous variables. A paired $t$ test was used to compare the change from baseline to the different follow-up time values for each group separately. Differences of categorical data were analyzed by using chi-square and Fisher exact tests. Tests were considered significant if $P<0.05$.

\section{Results}

\subsection{Preoperative and Postoperative NDI Scores and JOA Scores}

Patients in both the BMP group and IBG group had comparable preoperative clinical data. (Table 1) All patients had a successful ACDF procedure. The surgical data is listed in Table 2. There was no statistical 
difference for the duration of the operation for two-level ACDF between two groups $(P>0.05)$, while the duration of the operation for one-level ACDF showed a statistical difference $(P<0.05)$. No difference was seen between groups for estimated blood loss (EBL) in one- and two-level ACDF $(P>0.05)$. There was statistically significant difference regarding hospital stay between two groups $(P=0.000)$. BMP group had a longer hospital stay days than IBG group.

NDI scores and JOA scores were recorded preoperatively and at every follow-up. There were no statistically significant differences for postoperative NDI scores and JOA scores between the BMP group and IBG group ( $P>0.05)$. However, the postoperative JOA scores and NDI scores in both groups had significant differences from the preoperative scores, respectively $(P<0.01)$. (Table 3$)$ All patients in both groups had a solid fusion at the 3-month follow-up according to dynamic lateral X-ray and CT scans.

\subsection{PSTS diameters and Dysphagia identifications}

PSTS diameters were compared between the two groups at different time intervals. In the IBG group, the PSTS at C3 level was $3.9 \pm 1.4 \mathrm{~mm}$ preoperatively and increased to $12.6 \pm 4.5 \mathrm{~mm}$ postoperatively on the third day; the difference was statistically significant $(P<0.01)$. The PSTS significantly decreased at the 1month follow-up $(5.7 \pm 1.9 \mathrm{~mm}, \mathrm{P}<0.01)$ and continued to decrease at $3-, 6$-, and 12 -month. The PSTS at C6 level significantly increased postoperatively, from $15.3 \pm 2.2 \mathrm{~mm}$ preoperative to $17.1 \pm 3.1 \mathrm{~mm}$ on the third day postoperative $(P<0.01)$ and decreased gradually at the follow-up time intervals. In the BMP group, the PSTS at C3 level was $4.1 \pm 1.4 \mathrm{~mm}$ preoperatively and increased to $16.9 \pm 6.3 \mathrm{~mm}$ postoperatively; the difference was statistically significant $(P<0.001)$. The PSTS significantly decreased at 1-month follow-up $(7.7 \pm 4.2 \mathrm{~mm}, \mathrm{P}<0.01)$ and continued to decrease at 3-, 6-, and 12-month. The PSTS at C6 level significantly increased postoperatively, from $15.6 \pm 2.6 \mathrm{~mm}$ preoperative to $20.7 \pm 3.7 \mathrm{~mm}$ postoperative $(P<0.01)$ and decreased gradually at 1-, 3-, 6- and 12-month follow-ups time intervals. (Table 4)

The diameter of PSTS was compared between the two groups at each time interval. Both C3 and C6 level PSTS diameters had no statistically differences at preoperative time intervals ( $p>0.05)$. There was a statistically significant difference regarding C3 level PSTS between BMP and IBG group at each postoperative time intervals $(P<0.01)$. PSTS at C3 level was more severe in the BMP group than in the IBG group. PSTS at C6 level had a statistically significant difference between the two groups at postoperative third day $(P<0.01)$ and postoperative 3-month $(0.01<P<0.05)$, while no statistically differences were seen at postoperative 1-, 6- and 12-month $(P>0.05)$.

In the BMP group, 2 patients (both 2-level ACDF) had a mild dysphagia, 1 patient (2-level ACDF) had a moderate dysphagia. The total dysphagia rate was $2.7 \%$. In the IBG group, 1 patient had a mild dysphagia (2-level ACDF). The total dysphagia rate was $1.6 \%$. The symptoms gradually disappeared within 2 weeks, no additional treatment required. There was no statistical difference between the two groups for dysphagia rate $(P=0.759)$. (Table 5$)$.

No hematoma/seroma and infection occurred in both groups. In the BMP group, except one severe dysphagia case, another patient had one anchoring clip loosening of the ROI-C implantation. The patient 
was placed under observation because there were no clinical symptoms. In the IBG group, one patient had a lateral femoral cutaneous nerve injury which recovered 6 months postoperatively. No pseudarthrosis were noticed in both groups.

\section{Discussion}

\subsection{Brief review of former studies reveals issues in the field to be solved}

Although using iliac crest bone grafts (autograft) in ACDF have an excellent clinical outcome, there have been several studies indicating pain ranging from approximately $2 \%$ to $40 \%$ in IBG donor-site[ $[19,20]$. Other complications including hematoma, sensory abnormalities, infection and fracture[21]. Additionally, using allograft bone has shown greater nonunion rates which could lead to additional surgery [9].

Stark et al [22] systematically reviewed 22 published articles about using a bone graft substitute in oneor two-level ACDF. The results indicated all studies using BMP showed 100\% fusion rate, though dysphagia was significantly greater. Frenekel et al [23]reported patients had no complication in rhBMP-2 low dosage (< $0.5 \mathrm{mg} /$ level), but reported $12.5 \%$ ( 1 patient) in middle dose $(0.5-1.1 \mathrm{mg} / \mathrm{level}$ ) and $50 \%$ (4 patients) in high dose (1.4-2.1 mg/level), showing the increasing dose-dependent complication rate.

Since the FDA released the warning, the proportion of using BMP in ACDF has significantly decreased [24]. However, the number of patients using BMP remained relatively stable [25]. The main concern is prevertebral soft tissue swelling and dysphagia which could cause airway compromise. Lu et al [26] reported the use of rhBMP-2 for two-level ACDF significantly increases the severity of dysphagia but did not affect the overall incidence of dysphagia. However, no statistically significant difference for the incidence or severity of dysphagia was found between patients undergoing three or four level ACDF treated with PEEK/rhBMP-2 and those treated with only allograft. Another study reported that high-dose rhBMP-2 $(2.1 \mathrm{mg} /$ level) is used in each fusion segment of cervical anterior fusion surgery, and $23.2 \%$ of patients experience hematoma, extensive pre-neck edema, dysphagia or other neck complications [27]. In a retrospective cohort study of anterior cervical fusion surgery [23], the authors indicated that the complications of using BMP appeared to be dose-related and of low incidence when the usage of BMP is equal to or less than $1.1 \mathrm{mg} /$ level. Tumialán et al [28] retrospectively reviewed 200 patients who underwent a single- or multilevel ACDF. The results showed the incidence of symptomatic dysphagia may be decreased with a lower dose of rhBMP-2. In single- and 2-level fusions, in which the total dose of rhBMP-2 was $0.7-1.05 \mathrm{mg}$ and $1.4-2.10 \mathrm{mg}$, respectively, the resulting incidence of dysphagia was 1 and $3.2 \%$, respectively.

How to reduce the incidence and severity of prevertebral soft tissue swelling (PSTS) and dysphagia are the most concerning question when rhBMP-2 is used in anterior cervical spine surgery. Perri et al [29] reported a 54-year-old male patient who had neck swelling and difficulty swallowing after ACDF using rhBMP-2; however, there was no mention of the dose of rhBMP-2 used. In addition, the patient had a 
notably significant immunodeficiency virus. They used a bioresorbable PLa cage which potentially could lead to adverse tissue reactions in spinal and other orthopedic surgeries. In addition to these potential factors, they also concluded that the dose and delivery/carrier of rhBMP-2 were related to adverse soft tissue inflammation. Edwards et al [30] prospectively investigated whether the local administration of depomedrol decreases the severity of dysphagia after ACDF using BMP. The results showed depomedrol decreased dysphagia incidences and magnitude at all time intervals, with significant statistical differences on postoperative days $4,7,14$, and $28(P<0.05)$. Schroeder et al [31] conducted a retrospective radiological study in patients who undergoing 2- or 3-level primary ACDF without BMP; they found a steroid-soaked gelatin sponge significantly reduced postoperative soft tissue swelling. Song et al [32] used methylprednisolone intravenously ( $250 \mathrm{mg}$ per 6 hours, only for 24 hours) after multilevel ACDF surgery in a prospective study.

There were no complications associated with methylprednisolone. However, the usage of steroids in patients undergoing ACDF remains controversial [33].

There were limitations in this study. First, implantation of ACDF procedure is not standard. Although polyetheretherketone (PEEK) cages were used in all patients, some patients had anterior cervical plates while others did not. Second, since multilevel ACDF is one of reasons for dysphagia [34], we did not analyze rhBMP-2 usage in more than two levels ACDF. Third, we did not compare the cost of using rhBMP-2 graft with harvesting iliac crest bone graft and allograft bone graft.

\subsection{Summary of current study provides promising results and solutions}

Current study set a low dose of rhBMP-2 (0.5 mg/level) for ACDF (one- or two-level), which showed excellent clinical outcomes and a $100 \%$ solid fusion rate based on dynamic X-ray and CT scans at the 3month follow-up; and no pseudarthrosis occurrences. We used excised local osteophytes obtained from the decompression to cover the rhBMP-2 carrier bilaterally as an autograft and to mitigate the speed of release of rhBMP-2. The results showed that there were no additional surgeries and intubation related to use of rhBMP-2. The severity of dysphagia and PSTS were significantly diminished without any adverse effects. In our study, we used methylprednisolone intravenously to diminish PSTS and dysphagia perioperatively. The total dysphagia rate in the BMP group was $2.7 \%$ (3 in 110 patients). The complications due to of rhBMP-2 was rare and no severe adverse event lasted. Although the stay of hospital days was longer in BMP group than IBG group, the clinical outcome (NDI and JOA score) has no statistically difference. The main reason of prolonged hospitalization was to anti-swelling therapy and to require a diet to sustain adequate nutrition. The carrier of the rhBMP-2 was hydroxyapatite (HA), injectable lecithin and collagen sponge. It was different with INFUSE (Medtronic Sofamor-Danek, Memphis, TN) which used absorbable collagen sponge (ACS) as the carrier. The complex carrier has several advantages, such as, adsorption and sustained release of rhBMP-2, providing cell growth matrix, biocompatibility, no immune reaction and certain bone conductivity. It provides excellent physiological 
support for the deposition and regeneration of new bone and can form a direct bony bond with the bone tissue.

\section{Conclusions}

In conclusion, the clinical outcome of low dose rhBMP-2 $(0.5 \mathrm{mg} / \mathrm{level})$ for one- and two-level ACDF is excellent. It can reach $100 \%$ solid fusion at 3 months which is comparable with iliac crest bone graft, while avoiding autograft donor-site complications. The adverse events associated with rhBMP-2, such as severe soft tissue swelling, dysphagia, hematoma/seroma and excess bone formation are rare. We recommend placing the rhBMP-2 carrier in the center of cage, and bilateral coverage of the carrier with excised local osteophytes to decrease the speed of release of rhBMP-2.

\section{Declarations}

\section{Ethics approval and consent to participate}

The study protocol was established, according to the ethical guidelines of the Helsinki Declaration and was approved by the Human Ethics Committee of First Affiliated Hospital of Soochow University. Written informed consent was obtained from individual or guardian participants.

\section{Consent to publish}

Not applicable.

\section{Availability of data and materials}

Not applicable.

\section{Competing interests}

The authors declare that they have no competing interests

\section{Funding}

This study was supported in part by grants from Jiangsu Provincial Special Program of Medical Science 『BL2012004)

\section{Authors' contributions}


JWM supervised all aspects of the project. SJH,YHL and ZXS made substantial contributions to the conception and study design of this manuscript. SJH drafted the manuscript. HYH,SS,YF,LG,BJ, SM,ZF and JWM performed ACDF procedure as well as critically reviewed the manuscript and provided their comments. All authors reviewed the manuscript and provided their consent for publication. All authors read and approved the final manuscript.

\section{Acknowledgements}

We appreciated Dr. Yijie Liu and Dr. Heng Wang for data collection for the manuscript.

\section{References}

1.Wozney, J. M., V. Rosen, A. J. Celeste, et al., (1988) Novel regulators of bone formation: molecular clones and activities. Science, 242(4885): p. 1528-34.

2.Wozney, J. M., (1989) Bone morphogenetic proteins. Prog Growth Factor Res, 1(4): p. 267-80.

3.Burkus, J. K., S. E. Heim, M. F. Gornet, et al., (2004) The effectiveness of rhBMP-2 in replacing autograft: an integrated analysis of three human spine studies. Orthopedics, 27(7): p. 723-8.

4.Boden, S. D., J. Kang, H. Sandhu, et al., (2002) Use of recombinant human bone morphogenetic protein-2 to achieve posterolateral lumbar spine fusion in humans: a prospective, randomized clinical pilot trial: 2002 Volvo Award in clinical studies. Spine (Phila Pa 1976), 27(23): p. 2662-73.

5.Vaidya, R., J. Carp, A. Sethi, et al., (2007) Complications of anterior cervical discectomy and fusion using recombinant human bone morphogenetic protein-2. Eur Spine J, 16(8): p. 1257-65.

6.Carragee, E. J., E. L. Hurwitz, and B. K. Weiner, (2011) A critical review of recombinant human bone morphogenetic protein-2 trials in spinal surgery: emerging safety concerns and lessons learned. Spine J, 11(6): p. 471-91.

7.Mroz, T. E., J. C. Wang, R. Hashimoto, et al., (2010) Complications related to osteobiologics use in spine surgery: a systematic review. Spine (Phila Pa 1976), 35(9 Suppl): p. S86-104.

8.Floyd, T. and D. Ohnmeiss, (2000) A meta-analysis of autograft versus allograft in anterior cervical fusion. Eur Spine J, 9(5): p. 398-403.

9.Suchomel, P., P. Barsa, P. Buchvald, et al., (2004) Autologous versus allogenic bone grafts in instrumented anterior cervical discectomy and fusion: a prospective study with respect to bone union pattern. Eur Spine J, 13(6): p. 510-5.

10.Bishop, R. C., K. A. Moore, and M. N. Hadley, (1996) Anterior cervical interbody fusion using autogeneic and allogeneic bone graft substrate: a prospective comparative analysis. J Neurosurg, 85(2): p. 206-10. 
11.Bazaz, R., M. J. Lee, and J. U. Yoo, (2002) Incidence of dysphagia after anterior cervical spine surgery: a prospective study. Spine (Phila Pa 1976), 27(22): p. 2453-8.

12.Lee, S. H., K. T. Kim, K. S. Suk, et al., (2011) Effect of retropharyngeal steroid on prevertebral soft tissue swelling following anterior cervical discectomy and fusion: a prospective, randomized study. Spine (Phila Pa 1976), 36(26): p. 2286-92.

13.Lee, M. J., R. Bazaz, C. G. Furey, et al., (2007) Risk factors for dysphagia after anterior cervical spine surgery: a two-year prospective cohort study. Spine J, 7(2): p. 141-7.

14.Lee, M. J., R. Bazaz, C. G. Furey, et al., (2005) Influence of anterior cervical plate design on Dysphagia: a 2-year prospective longitudinal follow-up study. J Spinal Disord Tech, 18(5): p. 406-9.

15.Kim, S. W., C. Jang, M. H. Yang, et al., (2017) The natural course of prevertebral soft tissue swelling after anterior cervical spine surgery: how long will it last? Spine J, 17(9): p. 1297-1309.

16.Riew, K. D., J. J. Yang, D. G. Chang, et al., (2018) What is the most accurate radiographic criterion to determine anterior cervical fusion? Spine $J$.

17.Audat, Z. A., M. D. Fawareh, A.M. Radydeh, et al., (2018) Anterior versus posterior approach to treat cervical spondylotic myelopathy, clinical and radiological results with long period of follow-up. SAGE Open Med, 6: p. 2050312118766199.

18.Zhang, B., S. Li, D. Miao, et al., (2018) Risk Factors of Cage Subsidence in Patients with Ossification of Posterior Longitudinal Ligament (OPLL) After Anterior Cervical Discectomy and Fusion. Med Sci Monit, 24: p. 4753-4759.

19.Sasso, R. C., J. C. LeHuec, and C. Shaffrey, (2005) Iliac crest bone graft donor site pain after anterior lumbar interbody fusion: a prospective patient satisfaction outcome assessment. J Spinal Disord Tech, 18 Suppl: p. S77-81.

20.Myeroff, C. and M. Archdeacon, (2011) Autogenous bone graft: donor sites and techniques. J Bone Joint Surg Am, 93(23): p. 2227-36.

21.Fasolis, M., P. Boffano, and G. Ramieri, (2012) Morbidity associated with anterior iliac crest bone graft. Oral Surg Oral Med Oral Pathol Oral Radiol, 114(5): p. 586-91.

22.Stark, J. R., J. Hsieh, and D. Waller, (2018) Bone Graft Substitutes in Single or Double Level Anterior Cervical Discectomy and Fusion: A Systematic Review. Spine (Phila Pa 1976).

23.Frenkel, M. B., K. S. Cahill, R. J. Javahary, et al., (2013) Fusion rates in multilevel, instrumented anterior cervical fusion for degenerative disease with and without the use of bone morphogenetic protein. $J$ Neurosurg Spine, 18(3): p. 269-73. 
24.Lord, E. L., J. R. Cohen, Z. Buser, et al., (2017) Trends, Costs, and Complications of Anterior Cervical Discectomy and Fusion With and Without Bone Morphogenetic Protein in the United States Medicare Population. Global Spine J, 7(7): p. 603-608.

25.Cole, T., A. Veeravagu, B. Jiang, et al., (2014) Usage of recombinant human bone morphogenetic protein in cervical spine procedures: analysis of the MarketScan longitudinal database. J Bone Joint Surg Am, 96(17): p. 1409-16.

26.Lu, D.C., L. M. Tumialan, and D. Chou, (2013) Multilevel anterior cervical discectomy and fusion with and without rhBMP-2: a comparison of dysphagia rates and outcomes in 150 patients. J Neurosurg Spine, 18(1): p. 43-9.

27.Shields, L. B., G. H. Raque, S. D. Glassman, et al., (2006) Adverse effects associated with high-dose recombinant human bone morphogenetic protein-2 use in anterior cervical spine fusion. Spine (Phila Pa 1976), 31(5): p. 542-7.

28.Tumialan, L. M., J. Pan, G. E. Rodts, et al., (2008) The safety and efficacy of anterior cervical discectomy and fusion with polyetheretherketone spacer and recombinant human bone morphogenetic protein-2: a review of 200 patients. J Neurosurg Spine, 8(6): p. 529-35.

29.Perri, B., M. Cooper, C. Lauryssen, et al., (2007) Adverse swelling associated with use of rh-BMP-2 in anterior cervical discectomy and fusion: a case study. Spine J, 7(2): p. 235-9.

30.Edwards, C. C., 2nd, C. Dean, C. C. Edwards, et al., (2016) Can Dysphagia Following Anterior Cervical Fusions With rhBMP-2 Be Reduced With Local Depomedrol Application?: A Prospective, Randomized, Placebo-Controlled, Double-Blind Trial. Spine (Phila Pa 1976), 41(7): p. 555-62.

31.Schroeder, J., J. Weinstein, S. N. Salzmann, et al., (2018) Effect of Steroid-Soaked Gelatin Sponge on Soft Tissue Swelling Following Anterior Cervical Discectomy and Fusion: A Radiographic Analysis. Asian Spine J, 12(4): p. 656-661.

32.Song, K. J., S. K. Lee, J. H. Ko, et al., (2014) The clinical efficacy of short-term steroid treatment in multilevel anterior cervical arthrodesis. Spine J, 14(12): p. 2954-8.

33.Siasios, I., K. Fountas, V. Dimopoulos, et al., (2018) The role of steroid administration in the management of dysphagia in anterior cervical procedures. Neurosurg Rev, 41(1): p. 47-53.

34.Wu, B., F. Song, and S. Zhu, (2017) Reasons of Dysphagia After Operation of Anterior Cervical Decompression and Fusion. Clin Spine Surg, 30(5): p. E554-e559.

\section{Figures}




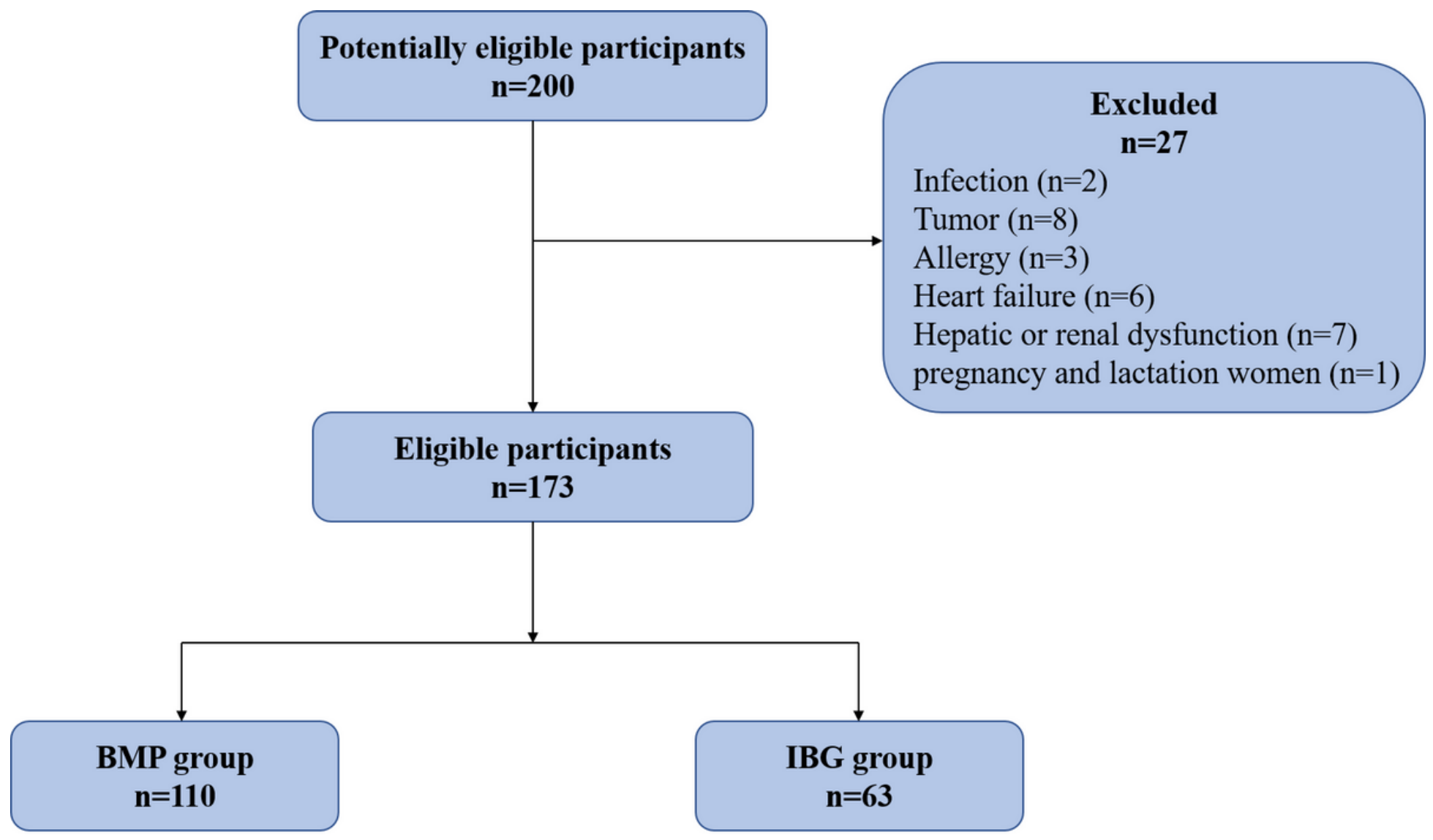

\section{Figure 1}

Flowchart showing process of patient selections.

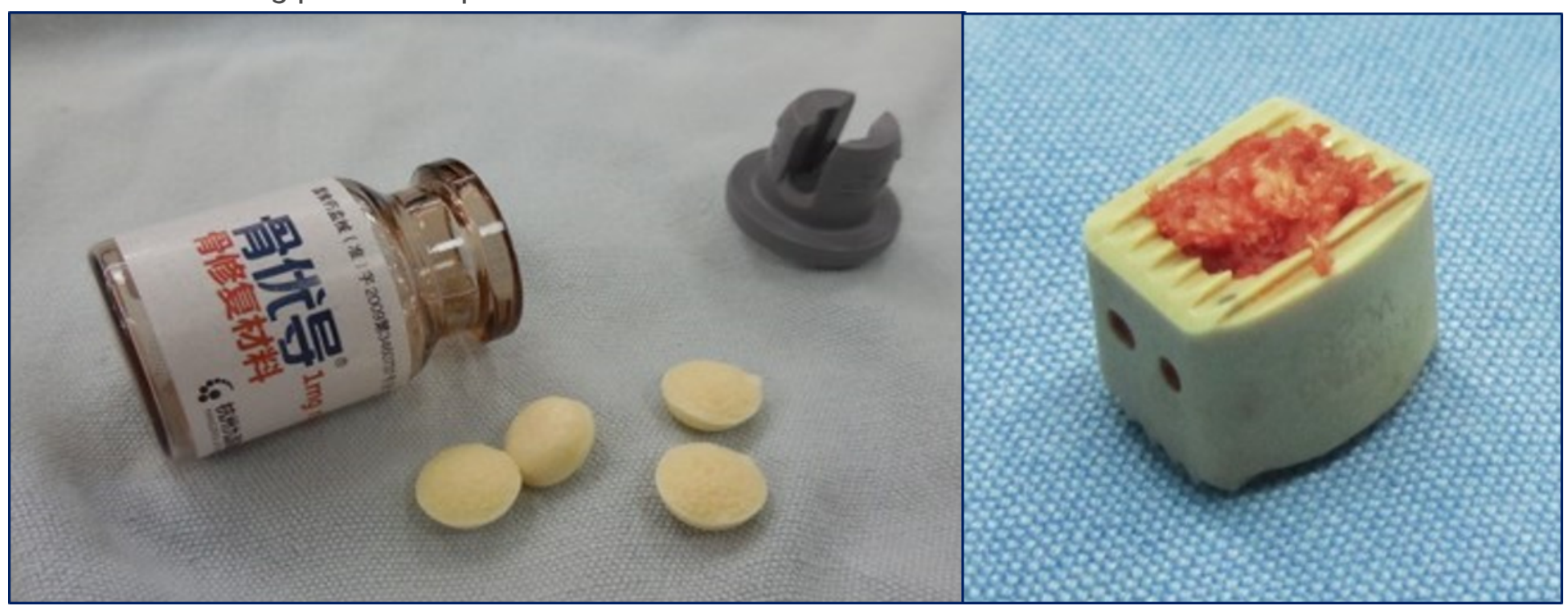

Figure 2

a. rhBMP-2 applied to an absorbable collagen sponge composited with hydroxyapatite and injectable lecithin. Each carrier contains $0.25 \mathrm{mg}$ rhBMP-2. b. During the ACDF procedure, the rhBMP-2 carriers were 
placed in the center of cage, the excised local osteophytes obtained from the decompression covered the carriers bilaterally.

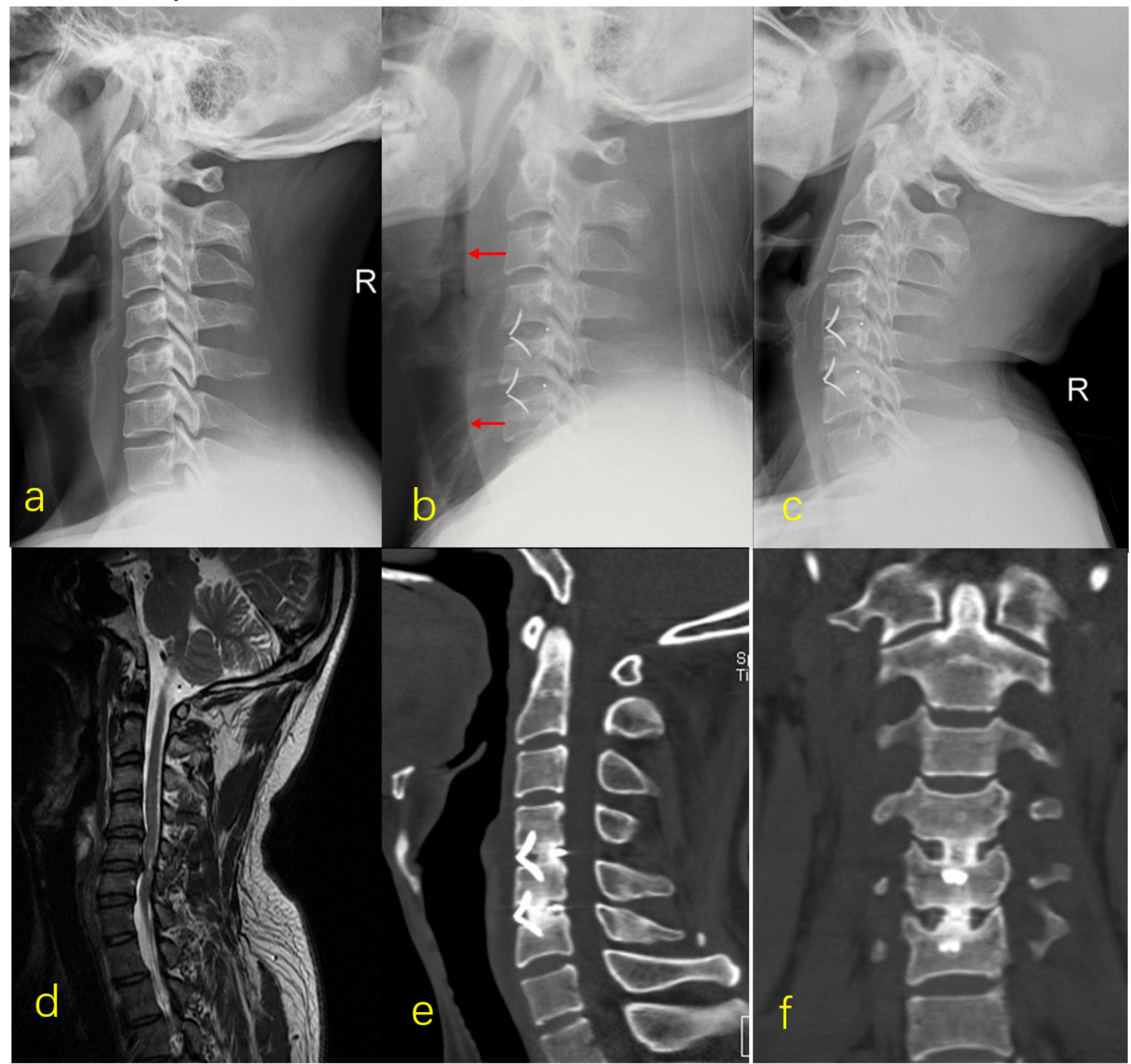

\section{Figure 3}

a. Pre-operative lateral X-ray. b. Post-operative third day, PSTS was measured at C3 and C6 level. c. Postoperative 3 months, $X$-ray showed a continuous bone bridge between the intervertebral bodies. $d$. Preoperative MRI. e and f. Post-operative 3 months, CT showed solid fusion as well as no excess bone formation. 